Rabaska

Revue d'ethnologie de l'Amérique française

\title{
Présence de Marius Barbeau
}

Jean-François Blanchette

Volume 14, 2016

URI : https://id.erudit.org/iderudit/1037462ar

DOI : https://doi.org/10.7202/1037462ar

Aller au sommaire du numéro

Éditeur(s)

Société québécoise d'ethnologie

ISSN

1703-7433 (imprimé)

1916-7350 (numérique)

Découvrir la revue

Citer ce document

Blanchette, J.-F. (2016). Présence de Marius Barbeau. Rabaska, 14, 208-212.

https://doi.org/10.7202/1037462ar d'utilisation que vous pouvez consulter en ligne.

https://apropos.erudit.org/fr/usagers/politique-dutilisation/ 


\section{Colloques et rassemblements}

\section{Présence de Marius Barbeau}

Jean-François Blanchette Chercheur associé au Musée canadien de l'histoire

À l'occasion du centenaire du premier terrain de Marius Barbeau (18831969) sur les Canadiens français, quarante spécialistes, en provenance du Québec, de l'Ontario, de la France et des États-Unis, ont participé au colloque international qui s'est tenu du 2 au 5 octobre 2014 au Domaine Forget en Charlevoix ; il avait pour titre : Présence de Marius Barbeau - L'invention du terrain en Amérique française - Autour d'un legs centenaire (1914-2014). L'objectif premier était la célébration de la fondation de leur champ disciplinaire, l'ethnologie des francophones d'Amérique avec le terrain comme base de la recherche. Ils désiraient également évaluer la méthode de travail, les analyses, les synthèses et les conclusions du fondateur. Comme ce colloque a eu lieu deux générations après la fin des travaux de Barbeau, il fut possible de regarder le tout avec un œil critique nouveau. Car il s'avère important de réaliser le chemin parcouru en un siècle, depuis ce premier terrain de l'été 1914 jusqu'à ce colloque de l'automne 2014.

Ces spécialistes sont venus présenter les résultats de leurs recherches dans les champs de la littérature orale, de la musique, de la culture matérielle, sans oublier les méthodes de recherche sur le terrain, les techniques d'archivage et leur interprétation, en référence à l'influence de Marius Barbeau sur leurs travaux. Dix séances ont regroupé les communications et échanges des tables rondes sous les thèmes Terrain et territoire - le Pays des gourganes ; Terrain et territoire - Barbeau et l'oralité; Barbeau et l'oralité : la chanson; Barbeau, promoteur de la tradition; Nos traditions à l'Université; Musées, muséologie, archives ; Formation et statut du chercheur ; L'apport de Barbeau aux arts religieux et populaires; Infuences diverses; Barbeau et le terrain : un exemple pour demain? Le colloque a été clôturé par l'allocution de JeanPierre Pichette, président de la Société québécoise d'ethnologie, «Barbeau : au-delà du souvenir » et par une visite de lieux patrimoniaux locaux. 
Ce colloque international fut organisé par la Société québécoise d'ethnologie $(\mathrm{S} Q \mathrm{E})$, la Société d'histoire de Charlevoix et le Centre de recherche sur l'histoire et le patrimoine de Charlevoix en partenariat avec la Chaire de recherche du Canada en oralité des francophonies minoritaires d'Amérique (COFRAM - Université Sainte-Anne). Le lecteur pourra consulter le programme du colloque ainsi que le résumé des communications sur le site web de la $\mathrm{SQE}^{1}$. De plus, les textes des communications ont été publiés comme volume 13 (2015) de la revue Rabaska ${ }^{2}$. Par conséquent, ce compte rendu sera bref. Mes commentaires ne toucheront pas toutes les communications, mais certaines d'entre elles qui me permettront d'illustrer la tangente de mon propos dans lequel je désire faire écho à l'observation et à la critique apportées par certains conférenciers sur l'approche de terrain de Barbeau et certaines de ses conclusions. Car la maturité de la discipline de même que les recherches nouvelles autorisent les chercheurs à proposer des interprétations différentes que celles auxquelles Barbeau en était arrivé.

La circonstance de ce colloque était la célébration des débuts des travaux de terrain de Marius Barbeau en ethnologie canadienne-française. En effet, il y a cent ans, Marius Barbeau commençait ses études de terrain dans la région de Charlevoix et posait sans s'en douter le premier jalon de ce qui allait devenir la pierre angulaire des études ethnologiques des Francophones d'Amérique. Pendant un demi-siècle il allait poursuivre ses travaux et travailler ardemment à les diffuser au moyen d'activités de communications de toutes sortes, conférences, expositions, festivals, articles dans des revues populaires et savantes, de nombreuses publications pour tous les publics, des entrevues radiophoniques et même des films. Il collectionnera aussi des objets pour les institutions culturelles du pays et d'ailleurs, mais en particulier pour le Musée national du Canada qui l'avait engagé. Tout ce matériel recueilli par Barbeau allait servir à notre connaissance du patrimoine en même temps qu'il ouvrait de nombreuses avenues de recherche dans tous les secteurs de l'ethnologie, comme en témoignent les spécialistes conférenciers de ce colloque.

Marius Barbeau a une vision précise qui le guide dans tous ses travaux : la recherche et la documentation des effets de la présence française et de son adaptation en terre d'Amérique. Jean-Pierre Pichette dans son analyse de l'impact de l'œuvre fondatrice de Barbeau, Le Romancero du Canada indique : "On voit ainsi comment la substance du livre, son ordonnance thématique et le contenu des commentaires mettent à jour les intuitions profondes et les positions théoriques qui animent l'auteur, en particulier sur l'origine française et la haute qualité de la tradition canadienne. » Car c'est cela qui non seulement guide le chercheur, mais le motive et l'oriente. C'est

1. Voir ethnologiequebec.org/2015/01/colloque-marius-barbeau-compte-rendu-et-resumes-descommunications.

2. Ouvrage disponible au bureau de la SQE. 
cette dynamique qui caractérise les travaux de Barbeau tout au long de sa carrière. Son analyse des réalités observées va par conséquent être teintée par cette préoccupation intellectuelle comme plusieurs exposés de ce colloque nous le révèlent. Car, après avoir louangé les travaux du fondateur de l'ethnologie canadienne-française, il est de mise, deux générations après la fin de ses travaux, de réexaminer ses méthodes de terrain, les données qu'il a recueillies, ses analyses de même que ses interprétations et ses conclusions.

Amelia-Elena Apetrei indique que Barbeau voyait dans les contes recueillis au Québec " le parler français ancestral pur et intact » alors que certaines analyses récentes montrent que la structure et les motifs des contes ont plus de liens avec les contes des coureurs de bois qui les ont adoptés et modifiés. Dans le champ de la chanson traditionnelle, Marcel Bénéteau montre l'effet que l'approche de Barbeau, qui visait à retracer les chansons françaises originales, a eu sur la transmission des chansons par la suite, par exemple sur les rives de la rivière Détroit. Il indique que Barbeau voulait que les chanteurs de son époque utilisent les versions les plus anciennes, celles qui étaient les plus près des versions venues de France avec les immigrants aux $\mathrm{XVII}^{\mathrm{e}}$ et $\mathrm{XVIII}^{\mathrm{e}}$ siècles. Barbeau a une approche similaire en culture matérielle quand il favorise les travaux du sculpteur sur bois Médard Bourgault au détriment de ses frères Jean-Julien et André qui, à ses yeux, s'étaient éloignés de la tradition française en innovant et en développant un autre style. C'est la conclusion à laquelle en vient Jean-François Blanchette qui présente l'opposition entre d'une part la vision de Barbeau qui recherchait l'authenticité des traditions de la culture matérielle et leur origine en Nouvelle-France et d'autre part la vision des modernistes comme Jean-Marie Gauvreau qui proposaient de réorienter le savoir-faire des artisans vers un nouvel esthétique plus conforme aux marchés en temps de crise économique, entre 1929 et 1945.

Diane Joly fait état du développement de la pensé de Barbeau en utilisant la correspondance entre celui-ci et Édouard-Zotique Massicotte : «D’abord empreinte de sollicitude, la correspondance révèle peu à peu leur vision du folklore où se confrontent les idées. À la suite d'échanges rudes, ils cessent de collaborer en 1921 et leurs écrits s'espacent par la suite. » Car Barbeau voit dans le folklore un outil pour combattre la modernité. En effet, l'infatigable Marius Barbeau se sentait interpelé par la perte des traditions dans les champs de l'ethnologie matérielle comme immatérielle devant les avancées de la modernité sur les modes de vie. Marilie Labonté qui examine la contribution de Barbeau à la muséologie raconte que Barbeau n'aimait pas la radio et la télévision qui à ses yeux corrompaient la culture traditionnelle, mais il les utilisera tout de même.

Dans le domaine de la culture matérielle toujours, Louise Lalonger indique que les notes prises par Barbeau entre 1911 et 1940 témoignent de 
son intérêt pour les teintures domestiques au moyen de colorants indigènes, de colorants naturels importés ou de produits synthétiques. Il est clair que Barbeau désire documenter l'ancienneté des techniques utilisées en cherchant à identifier les teintures des textiles qu'il a acquis. Mais Lalonger fait des mises en garde contre les interprétations que les ethnologues comme Barbeau et Robert-Lionel Séguin ont faites sur l'identification des teintures utilisées. Car l'analyse en laboratoire de textiles anciens a révélé des résultats différents de ce que les ethnologues avaient consignés, les teintures synthétiques étant plus courantes. En effet, l'examen en laboratoire devrait toujours être complémentaire des observations faites par les ethnologues et des informations reçues de leurs informateurs. Il appert en effet que les teintures synthétiques ont envahi le marché, au Québec comme ailleurs, à la campagne comme à la ville, dès la fin du XIX ${ }^{e}$ siècle. Peut-on extrapoler et penser que l'approche de Barbeau dans ses terrains, comme nous l'a déjà révélé Benoît Thériault, ait eu un effet d'entraînement sur ses informateurs. En effet, Thériault a conté comment Barbeau suggérait à ses étudiants d'approcher leurs informateurs avec des questions directes, car ni les uns ni les autres n'avaient de temps à perdre. Vous leur dites : voici ce que je cherche, en avez-vous $?^{3}$ Cette approche comporte certaines difficultés, car les informateurs qui ne connaissent pas l'enquêteur veulent le satisfaire et lui donner ce qu'il désire, ce qui est bien naturel chez l'habitant qui désire plaire à l'étranger qui achète ses produits. De plus l'analyse à vue des teintures n'est pas évidente comme le rapporte Lalonger. C'est ainsi que Barbeau se retrouve avec des textiles qui, malgré ce qu'il croit, ne sont pas teints avec des teintures naturelles, autochtones ou importées, mais bien avec des teintures synthétiques.

La méthodologie de recherche sur le terrain a été traitée par plusieurs conférenciers. Barbeau documente ses terrains, choisit méticuleusement ses informateurs et les interroge patiemment, souvent à répétition si l'information est abondante. Christian Harvey montre comment Barbeau choisissait méticuleusement les endroits où il ferait du terrain, par exemple dans ces rangs de l'arrière-pays de Charlevoix, autour des Éboulements, car c'est là qu'il croit pouvoir trouver les traces les plus anciennes de traditions orales françaises de l'époque de la Nouvelle-France. En effet, l'objectif de Barbeau n'est pas de documenter tous les contes de la région, mais bien ceux qui, à ses yeux, se rattachent à cette période française.

En terminant, il faut mentionner que la majorité des communications de ce colloque ont porté sur les enquêtes de terrain et les documents du fonds Marius-Barbeau du Musée canadien de l'histoire, autrefois le Musée canadien

3. Marius Barbeau, « Comment on recueille les contes, les chants, les mélodies, le langage, les spécimens ou les données s’y rapportant, parmi les Sauvages ou les Blancs », présenté par Benoît Thériault dans « Marius Barbeau, en quête de connaissances... depuis 1911 », Rabaska, Revue d'ethnologie de l'Amérique française, vol. 9, 2011, p. 175. 
des civilisations. Ce fonds est impressionnant et sa consultation est facilitée par le travail coopératif de son archiviste Benoît Thériault, sans aucun doute la référence la plus utilisée au cours de ce colloque, outre celle de Marius Barbeau elle-même. Par ailleurs, aucun chercheur n'a fait mention du fonds Marius Barbeau conservé à la Bibliothèque et aux archives nationales du Québec à Montréal ${ }^{4}$. Ce fonds comprend de nombreux documents, manuscrits et copies de manuscrits, des chansons traditionnelles, de la correspondance et des photographies de Marius Barbeau légués à l'institution par son épouse après son décès. La matière qu'on y trouve est en rapport avec les travaux professionnels de Barbeau, mais également avec son enfance et sa vie privée. Ce fonds mériterait d'être consulté et utilisé dans les recherches futures sur le travail de Marius Barbeau et pour une compréhension plus complète de l'homme.

4. Fonds Marius-Barbeau, P51, Bibliothèque et archives nationales du Québec à Montréal : 3,36 mètres de documents, environ 3000 photographies, environ 200 gravures, 2 cartes, 4 vidéos, et un autre document. 